\title{
An ionising radiation-induced specific transcriptional signature of inflammation- associated genes in human whole blood: a pilot study
}

Lourdes Cruz-Garcia ( $\square$ lourdes.cruzgarcia@phe.gov.uk)

Public Health England https://orcid.org/0000-0002-7804-974X

Christophe Badie

Public Health England

Selvakumar Anbalagan

Institute of Cancer Research Sutton

Jayne Moquet

Public Health England

Lone Gothard

Institute of Cancer Research Sutton

Grainne O'Brien

Public Health England

Navita Somaiah

Institute of Cancer Research Sutton

Elizabeth A. Ainsbury

Public Health England

\section{Research}

Keywords: Ionising radiation, blood, gene expression, transcription, nCounter, qPCR, inflammation, Radiotherapy, cancer

Posted Date: January 25th, 2021

DOl: https://doi.org/10.21203/rs.3.rs-153147/v1

License: (c) (i) This work is licensed under a Creative Commons Attribution 4.0 International License. Read Full License 


\section{Abstract}

This communication reports the identification of a new panel of transcriptional changes in inflammationassociated genes observed in response to ionising radiation received by radiotherapy patients. Peripheral blood samples were taken with ethical approval and informed consent from a total of 20 patients undergoing external beam radiotherapy for breast, lung, gastrointestinal or genitourinary tumours. Nanostring nCounter analysis of transcriptional changes was carried out in samples prior and 24 hours post-delivery of the 1st radiotherapy fraction, just prior to the 5th or 6th fraction, and just before the last fraction. Statistical analysis with BRB Array Tools, GLM MANOVA and nSolver, revealed a radiation responsive panel of genes which varied by patient group (type of cancer) and with time since exposure (as an analogue for dose received), which may be useful as a biomarker of radiation response. Further validation in a wider group of patients is ongoing, together with work towards a full understanding of patient specific responses in support of personalised approaches to radiation medicine.

\section{Introduction}

A variety of different biological and physical retrospective tools are available to assess individual radiation doses following a radiation accident or incident (1-4). In recent years, transcriptional changes in blood have been identified as a promising biomarker for radiation response to support biodosimetric assessment of individual doses in accidental exposure scenarios $(5,6)$. The responsiveness of FDXR to ionising radiation at the transcriptional level in human blood was recently reported to provide accurate in vivo dose estimates and providing the first in vivo dose response in humans (7). Transcript variants of this gene have also shown a remarkable potential as standalone biomarkers for ionizing radiation exposure screenings (8). The influence of several potential confounding factors (cancer condition, sex, simulated bacterial infection (lipopolysaccharide), and curcumin, an anti-antioxidant agent) on radiation dose estimation using in vivo validated transcriptional biomarkers was also investigated, with the outcome that such confounding factors should not prevent the use of transcriptional responses for emergency triage purposes (9). Most recently, a new protocol for rapid gene expression-based dose estimation in human blood was reported (10), together with the generation of a transcriptional radiation exposure signature in human blood using long-read nanopore sequencing including several new genes representing ideal biomarkers of radiation exposure (11).

Although radiation-responsive genes such as FDXR have been reported in human white blood cells in radiotherapy patients, they have limited inter-individual variability in response; apart from early responsive genes such as CDKN1A which may be help to predict the severity of acute skin radiation toxicity (12), they are not informative of inter-individual variability in normal tissue sensitivity to radiation exposure. We previously reported that several genes associated with inflammatory processes (ARG1, BCL2L1, and MYC) present a long-term modification of transcriptional expression (13) towards the end of the radiotherapy treatment. In addition, there is strong evidence of radiation-induced inflammation feeding into innate and adaptive antigen-specific immune responses. This adds another dimension to the 
tumour-host crosstalk during radiation, in turn influencing normal tissue side effects following radiotherapy (14-18).

In this communication, we present a panel of inflammation-associated genes which are radiation responsive, validated in a population of radiotherapy patients, and shown to be differentially responsive for different patient groups and at different time points post-exposure.

\section{Methods}

The general conditions for ethical approval, patient selection and informed consent, blood sampling and individual patient dosimetry were described in detail in Moquet et al., (2018) (19). Briefly, blood samples from five breast, four endometrial, five lung, three prostate, two oesophagus and one colon cancer patients, treated with Intensity Modulated Radiotherapy (IMRT) using a linear accelerator (LINAC) were collected at four different time points during the course of the treatment: before the start of the treatment, $24 \mathrm{~h}$ after the first fraction, just before the fifth or sixth fraction and the last fraction. The prescribed doses for each patient were as follow: breast cancer patients received 40-40.5 Gy in 15 fractions, endometrial $45 \mathrm{~Gy}$ in 25 fractions, lung $55 \mathrm{~Gy}$ in 20 fractions, prostate $60 \mathrm{~Gy}$ in 20 fractions, oesophagus 36 in 12 fractions/20 Gy in 5 fractions and colon 40 Gy in 15 fractions. Patients did not receive previous radio- and/or chemotherapy treatments except for one of the lung cancer patients who received chemotherapy five weeks before the start of radiotherapy. Blood was collected at the Royal Marsden Hospital and Institute of Cancer Research (Surrey, UK) with written informed consent from all subjects as part of the RTGene study (ClinicalTrials.gov NCT02780375), which was ethically approved by the South Central-Hampshire B Research Ethics Committee (16/SC/0307).

\section{RNA isolation and reverse transcription}

Total RNA was extracted with the PAXgene Blood miRNA kit (Qiagen, PreAnalytiX GmbH, Hilden, Germany) using a robotic workstation Qiacube (Qiagen, Manchester, UK). The quantity of isolated RNA was determined by spectrophotometry with a ND-1000 NanoDrop and quality was assessed using a Tapestation 220 (Agilent Technologies, CA, USA). cDNA was prepared from $350 \mathrm{ng}$ of the total RNA using High Capacity cDNA reverse transcription kit (Applied Biosystems, FosterCity, CA, USA) according to the manufacturer's protocol.

\section{nCounter analysis}

Samples were analysed by the nCounter Analysis System (NanoString Technologies ${ }^{\circledR}$, Inc., Seattle, WA, USA) according to the manufacturers' guidelines. The samples were run using $100 \mathrm{ng}$ RNA per sample on the Human Inflammation V2 panel, which consists of 249 genes.

\section{Quantitative Real-time Polymerase Chain Reaction}

SYBRGreen qPCR was performed using Rotor-Gene Q (Qiagen, Hilden, Germany). All reactions were run in triplicate using PerfeCTa SYBR® Green SuperMix (Quanta Biosciences, Inc., Gaithersburg, MD, USA) with 
primer sets for target genes at $500 \mathrm{nM}$ concentration each. Cycling parameters were 2 min at $95^{\circ} \mathrm{C}$, then 40 cycles of $10 \mathrm{~s}$ at $95^{\circ} \mathrm{C}$ and $60 \mathrm{~s}$ at $60^{\circ} \mathrm{C}$. Data were collected and analysed by Rotor-Gene Q Series software. Fold of change values were calculated using the delta-delta Ct method (20). The primer sequences for SYBRGreen analysis were HPRT1 F: 5' TCAGGCAGTATAATCCAAAGATGGT 3', R: 5' AGTCTGGCTTATATCCAACACTTCG 3'; IL7 5' CTCCCCTGATCCTTGTTCTG 3', R: 5' TCATTATTCAGGCAATTGCTACC 3'; CD40LG F: 5' CACCCCCTGTTAACTGCCTA 3'; R: 3' CTGGATGTCTGCATCAGTGG 5'.

\section{BRBArrayTools and MANOVA}

Statistical analysis was performed with BRBArrayTools, to identify genes for which there were statistically significant changes (up or down regulation; $p<0.05$ ) associated with number of radiotherapy (RT) fractions and time since exposure, with a false discovery rate $(F D R)<0.05$. General Linear Model Multivariate Analysis of Variance (GLM ANOVA) and Multivariate Analysis of Variance (MANOVA) was then carried out with Minitab18 ${ }^{\circledR}$, to identify panels of genes significantly associated $(p<0.05)$ with radiation exposure, taking into account radiotherapy patient group by type of cancer treated (breast, lung, gastrointestinal or genitourinary tumours) and time since exposure (just before exposure, 24 hours postexposure, and just before the final fraction -range 3-5 weeks for all patients).

\section{Differential expression and pathway analysis}

Nanostring nCounter nSolver 4.0 (Nanostring Technologies) with the Advanced analysis plugging (version 2.0.134) was used to perform the differential expression (DE) and pathway analysis. DE analysis includes several multivariate linear regression models to identify significant genes (mixture negative binomial, simplified negative binomial, or log-linear model). FDR p-value adjustment was performed with Benjamini-Yekutieli method (21). Statistically significant, differentially expressed genes were defined as those with expression levels corresponding to a log2 ratio $>0.5$ or $<-0.5$ and $p$-value $<0.05$.

Gene set analysis (GSA) is a quantitative summary of DE for gene sets. Gene set's global significance score for a covariate is calculated as the square root of the mean squared t-statistic of genes. Global and directed significance scores were calculated for each pathway. Pathway scores were used to summarize data from a pathway's genes into a single score. Pathway scores were calculated as the first principal component of the pathway genes' normalized expression and standardized by $Z$ scaling. Pathway scoring helps to see how pathway scores change across samples. Increasing score corresponds to mostly increasing expression.

\section{Immune cell type profiling}

The cell type profiling module in Nanostring nCounter nSolver 4.0 advanced analysis was used to quantify cell populations using marker genes. Raw cell type measurements are calculated as the log2 expression of each cell type's marker genes and show the estimated abundances of each individual cell type between samples. 


\section{Results}

\section{BRBArrayTools and MANOVA}

A total of 29 genes were identified by BRB-ArrayTools as being significantly down or up regulated in response to ionising radiation exposure, with FDR $<0.05$. GLM ANOVA then revealed a subset of downregulated genes only for which both time since exposure and type of cancer were statistically significant, which indicates that these genes may be informative in understanding patient group specific responses. MANOVA on these genes reveals that this combined set of 7 genes (MYC, CD40LG, CCL4, IL7, TCF4, CCR7 and FASLG) is together statistically significantly reliant on both time post-exposure $(p=0.042)$ and cancer type $(p<0.001)$. For the up-regulated genes, no significant effects were identified for time postexposure.

\section{Differential Gene expression analysis}

DE analyses revealed a set of genes differentially expressed in blood samples at the time point before the last fraction (Figure 1). The genes TLR8, ALOX5, TYROBP, MAPK1, MYD8B, BCL6, HHGNI, MYC and MAPKAPK5 presented an up- or down-regulation ( $p$-vale<0.05) independently of the cancer type (Figure 1C). However, only MYC and BCL6 showed a fold change regulation above 1.5-fold (log2 FC >0.5 or < $-0.5)$.

\section{Gene set analysis (GSA)}

Differentially expressed gene sets were observed only when comparing the before time point with the last time point (before last fraction) (Figure 2A). These results highlighted the Interleukin 18 family and Class I MHC mediated antigen processing \& presentation genes, apoptosis, interleukin 20 family, platelet homeostasis and defensins pathways with the highest scores (Figure $2 \mathrm{~A}$ ). Directed global significance scores indicated that these pathways are upregulated at the last time point (Figure $2 \mathrm{~B}$ ).

\section{Pathway scoring}

Pathway score clustering showed a general separation of the time points for some of the cancer types, indicating higher scores at the last time point and also for breast cancer patients (Figure 3.A). When we compared pathway scores to time points (Figure 3B) we observed a decrease in NF-kB, cell cycle and apoptosis at the last time point. However, the scores in interleukin 1 signalling, Class I MHC mediated antigen processing \& presentation genes, cellular senescence, signalling by FGFR4, C-type lectin receptors and MAPK1MAPK3 signalling pathways were increased in the last time point (Figure 3B).

Regarding cancer type, interferon signalling pathway had a high score in breast cancer and low in colon and oesophagus compared to the other cancer types. Low scores were also observed for interleukin 1 signalling and metabolism pathways in colon. In contrast, the defensins pathway presented a high score in colon and oesophageal cancers. 
Pathway scoring between only the first and last time points showed two clusters one of higher scores in the last time points for a group of samples and a low score cluster for the first time point (before time point) which shows a switch on of several inflammation related pathways at the end of the radiotherapy treatment.

\section{Immune Cell profiling}

Cell type profiling analysis identify two main cell populations, macrophages and exhausted CD8+ T cells ( $T$ cells which adopt a functionally attenuated state due to prolonged antigen stimulation, characteristic of chronic infections and cancer). The average cell type score was compared between the different time points and first and last time points (Figure 4A). The results showed that macrophages were found to be relatively higher before the start of the treatment compared to the last time point but the opposite was found for the exhausted CD8 T cells (Figure 4A).

When we compared the cell type scores between the different cancer type (Figure 4B), the results revealed that breast and oesophagus cancer patients presented opposite changes in these two cell types compared to the other cancer types. Both cell types were higher in breast cancer patients but lower in oesophagus cancer patients compared to colon, prostate and lung cancers.

\section{Validation of nCounter analysis by $\mathrm{qPCR}$}

IL7 and CD40LG were selected to validate the nCounter analysis (Figure 5). The expression profiles of these genes were confirmed by qPCR with a significant down-regulation at the last time point (last fraction of the radiotherapy treatment).

\section{Discussion And Conclusions}

Our group has previously reported long-term modification of transcriptional expression in genes associated with inflammatory processes in head and neck and endometrial cancer patients undergoing radiotherapy (13).The aim of this study was to report, for the first time in a wide range of cancer types (breast, lung, prostate, endometrium and gastro-intestinal), details of a panel of inflammation-associated genes identified in radiotherapy patients as being significantly associated with ionising radiation exposure. Radiation responsive genes, both up and down regulated, were identified using a Human Inflammation V2 panel from nCounter Analysis system (NanoString Technologies ${ }^{\circledR}$ ) and assessed for the significance of their individual and combined responses in terms of time since exposure and type of cancer. The radiation doses received varied on a patient by patient basis (full data in Moquet et al., 2018 (19)), however, for the purposes of this initial analysis, as each time point pre- and post-exposure was at a different stage of the treatment, time post-exposure can be taken as an analogue for dose. Thus, the results of this work reveal gene sets and pathways which show significance in terms of radiation responsiveness for different groups of patients irrespective of the type of cancer. Both statistical approaches performed in this study identified a common radiation responsive gene, MYC. MYC is a protooncogene involved in cell cycle, cell proliferation, apoptosis (22), regulation of innate and adaptive host 
tumour immune responses (23) and it has been previously described as a radiation responsive gene in different cohorts (head and neck and endometrial cancer patients) (13). Only MYC and BCL6 were shown to be upregulated in our differential gene expression analysis performed with nSolver advanced analysis software, whereas the MANOVA analysis identified MYC, CD4OLG, CCL4, IL7, TCF4, CCR7 and FASLG as significantly differentially expressed. From those genes, CD40LG and IL-7 were observed to be significantly downregulated (before 5th -6th and last RT fraction) using nCounter analysis which was further confirmed by qPCR. However, in QPCR the significance of both CD40LG and IL-7 was observed only before last fraction among all the tumour types, demonstrating the sensitivity of these assays. CD40LG is transiently expressed on T cells as a result of inflammatory response and known to activate CD40 (24). CD40, a member of TNF family is known to be expressed by DC, myeloid cells and B cells and its activation leads to priming of cytotoxic T cells (25). Recently, IL-7 has been shown to be produced by radioresistant haematopoietic cells in mice (26). IL-7 regulates homeostasis of lymphocytes, survival and maintenance of T cells (27). These results suggest that CD4OLG and IL-7 have potential as immuneinflammatory markers to correlate dose fraction against volumes irradiated.

Pathway analysis revealed that there is a modulation of inflammation associated pathways after recurring exposure to radiation during the course of the radiotherapy treatment. It is known that IR can induce inflammation by inducing cytokines secretion and through bystander signals (28-30). GSA analysis highlighted two upregulated pathways at the last time point (before the last fraction), interleukin8 and class I MHC mediated antigen processing and presentation. Interleukin-18 is involved in activation and differentiation of various $T$ cell populations (31) and its increase has been linked to radiation injury (32). MHC class I peptides are antigens originated intracellular and delivered to the cell surface to be recognized by $\mathrm{CD} 8+T$ cells and an increase in this cell surface peptide presentation has been described after gamma irradiation exposure (33).

Looking at the individual cancer types, breast cancer patients presented a high score in the interferon pathway compared to the other type of cancers. Interferons are a family of cytokines which play an important role in initiating immune responses, especially antiviral and antitumour effects $(34,35)$. For colon and oesophageal cancers, defensins pathway presented a high score. The modulation of stress and multiple immune parameters were also reported for head and neck (36) and prostate adenocarcinomas (37) treated by radiotherapy.

Cell markers were present in the nCounter panel for macrophages and exhausted CD $8+T$ cells. These markers revealed changes in the levels of these cell types during the radiotherapy treatment and between cancer types. Continuous radiation exposure seems to promote a decrease in macrophages at the end of the treatment. Macrophage irradiation has demonstrated to modulate their phenotype towards a proinflammatory state promoting cancer angiogenesis and cancer cell-invasion (38). Macrophage activation and recruitment at site of injury has been proposed as an indirect effect of IR which results from cellular damage signals to clear radiation-induced apoptotic cells (28). This macrophage recruitment is in line with the slight decrease of macrophages in the early time point of the radiation therapy (24h after first fraction and before 5th -6th fraction). However, it is not clear why the macrophages decrease after long 
periods of repetitive exposures in the present study. When looking at individual cancer types, breast and oesophagus cancer patients differ in the level of macrophages compared to the other cancer types. Both oesophageal cancer patients had loco-regional lymph nodes included in the radiation field whereas only one out of 5 breast cancer patients had large radiotherapy volumes with lymph nodes irradiated. It is difficult to draw any firm biological conclusions given the small sample size, but this will be explored further in the ongoing follow-on study.

T cell exhaustion is an attenuated state of cell-response resulting from repeated or prolonged antigenic exposure under suboptimal conditions (39). So, it is not surprising to see this cell group increased after the continuous exposure to IR at the end of the radiotherapy treatment. T cell exhaustion also differs between different tumour types (40), as seen in the different cancer groups.

\section{Conclusion}

In summary, we confirmed that changes in expression of specific genes as well as systemic inflammatory responses showed great promise as markers for individual radiation dosimetry. Moreover, these results are encouraging and will be used as part of further research to understand individual radiation responses and explore the links between inflammatory and immune responses in the context of different dose fractionation schedules and volumes irradiated in various cancer types. Ultimately it is hoped these data will help further to develop personalized use of radiation in medicine.

\section{Abbreviations}

ARG1

Arginase 1

BCL2L1

Bcl-2-like protein 1

CCL4

C-C Motif Chemokine Ligand 4

CCR7

C-C chemokine receptor type 7

CD40LG

CD40 Ligand

FASLG

Fas Ligand

FDR

false discovery rate

FDXR

ferredoxin reductase

GSA

Gene set analysis 
HPRT1

Hypoxanthine-guanine phosphoribosyltransferase 1

IL7

Interleukin 7

IMRT

Intensity Modulated Radiotherapy

IR

ionizing radiation

LINAC

linear accelerator

MYC

MYC Proto-Oncogene

qPCR

Quantitative Real-time Polymerase Chain Reaction

RT

radiotherapy

TCF4

Transcription Factor 4

\section{Declarations}

\section{Ethics approval and consent to participate}

Blood was collected at the Royal Marsden Hospital and Institute of Cancer Research (Surrey, UK) with written informed consent from all subjects as part of the RTGene study (ClinicalTrials.gov NCT02780375), which was ethically approved by the South Central-Hampshire B Research Ethics Committee (16/SC/0307).

\section{Consent for publication}

Not applicable

\section{Availability of data and materials}

The datasets used and/or analysed during the current study are available from the corresponding author on reasonable request.

\section{Competing interests}

The authors declare that they have no competing interests

\section{Funding}


This work was partly supported by the National Institute for Health Research Health Protection Research Unit (NIHR HPRU) in Chemical \& Radiation Threats \& Hazards at Newcastle University in partnership with Public Health England (PHE) and partly funded by the NIHR HPRU in Chemical \& Radiation Threats \& Hazards a partnership between PHE and Imperial College London.. The views expressed are those of the author(s) and not necessarily those of the NIHR, PHE or the Department of Health and Social Care. The multi-panel coding and non-coding transcriptional responses as an indicator of individualized responses to radiation effects in radiation therapy patients, RTGene project, received a pilot grant from the Opportunity Funds Management Core of the Centers for Medical Countermeasures against Radiation, National Institute of Allergy and Infectious Diseases (Bethesda, MD) (grant no. U19AI067773) in collaboration with Columbia University (New York, NY). We acknowledge NHS funding to the NIHR Biomedical Research Centre at The Royal Marsden and ICR.

\section{Authors' contributions}

Conceptualization, C.B., E.A.A, N.S.; Writing-original draft, L.C.-G. and E.A.A.; Investigation, L.C.-G. and G.M.; Visualization, L.C.-G., Formal analysis, L.C.-G. and E.A.A; Supervision, C.B., E.A.A. and N.S., Recruiting patients and sample collection, S.A., J.M. and L.G.. Preparing grant applications, L.C.-G., G.M., C.B., E.A.A, N.S., S.A., J.M. and L.G.

\section{Acknowledgements}

The authors would like to thank all the patients and staff who participated in the study from the Royal Marsden NHS Foundation Trust, Sutton. In particular, we thank Drs. Fiona MacDonald (lung), Alison Tree (prostate), Susan Lalonrelle (endometrium), Diana Tait and Shree Bhide (gastrointestinal) for recruiting patients into this study. We are also very grateful to Ellen Donovan for input into RTGene study design and Sue Boyle for recruiting patients and managing this study at The Royal Marsden.

\section{References}

1. Rothkamm K, Beinke C, Romm H, Badie C, Balagurunathan Y, Barnard S. Comparison of established and emerging biodosimetry assays. Radiat Res. 2013;180.

2. Abend M, Badie C, Quintens R, Kriehuber R, Manning G, Macaeva E, et al. Examining RadiationInduced In Vivo and In Vitro Gene Expression Changes of the Peripheral Blood in Different Laboratories for Biodosimetry Purposes: First RENEB Gene Expression Study. Radiation Research. 2016;185(2):109-23.

3. Ainsbury E, Badie C, Barnard S, Manning G, Moquet J, Abend M, et al. Integration of new biological and physical retrospective dosimetry methods into EU emergency response plans - joint RENEB and EURADOS inter-laboratory comparisons. International Journal of Radiation Biology. 2017;93(1):99109.

4. Tichy A, Kabacik S, O’Brien G, Pejchal J, Sinkorova Z, Kmochova A, et al. The first in vivo multiparametric comparison of different radiation exposure biomarkers in human blood. PLOS ONE. 
2018;13(2):e0193412.

5. Badie C, Kabacik S, Balagurunathan Y, Bernard N, Brengues M, Faggioni G, et al. NATO BIODOSIMETRY STUDY: Laboratory Intercomparison of Gene Expression Assays. Radiation research. 2013;180(2):138-48.

6. Manning G, Macaeva E, Majewski M, Kriehuber R, Brzóska K, Abend M, et al. Comparable dose estimates of blinded whole blood samples are obtained independently of culture conditions and analytical approaches. Second RENEB gene expression study. International Journal of Radiation Biology. 2017a;93(1):87-98.

7. O’Brien G, Cruz-Garcia L, Majewski M, Grepl J, Abend M, Port M, et al. FDXR is a biomarker of radiation exposure in vivo. Scientific Reports. 2018;8(1):684.

8. Cruz-Garcia L, O’Brien G, Sipos B, Mayes S, Tichý A, Sirák I, et al. In Vivo Validation of Alternative FDXR Transcripts in Human Blood in Response to lonizing Radiation. International Journal of Molecular Sciences. 2020b;21(21).

9. Cruz-Garcia L, O’Brien G, Donovan E, Gothard L, Boyle S, Laval A, et al. Influence of Confounding Factors on Radiation Dose Estimation Using In Vivo Validated Transcriptional Biomarkers. Health Physics. 2018;115(1):90-101.

10. Polozov S, Cruz-Garcia L, Badie C. Rapid gene expression based dose estimation for radiological emergencies. Radiation Protection Dosimetry. 2019.

11. Cruz-Garcia L, O'Brien G, Sipos B, Mayes S, Love MI, Turner DJ, et al. Generation of a Transcriptional Radiation Exposure Signature in Human Blood Using Long-Read Nanopore Sequencing. Radiat Res. 2020a;193(2):143-54.

12. Badie C, Dziwura S, Raffy C, Tsigani T, Alsbeih G, Moody J, et al. Aberrant CDKN1A transcriptional response associates with abnormal sensitivity to radiation treatment. $\mathrm{Br} J$ Cancer. 2008;98(11):184551.

13. Manning G, Tichý A, Sirák I, Badie C. Radiotherapy-Associated Long-term Modification of Expression of the Inflammatory Biomarker Genes ARG1, BCL2L1, and MYC. Frontiers in Immunology. 2017b;8:412.

14. Schaue D, Micewicz ED, Ratikan JA, Xie MW, Cheng G, McBride WH. Radiation and inflammation. Semin Radiat Oncol. 2015;25(1):4-10.

15. Brandmaier A, Formenti SC. The Impact of Radiation Therapy on Innate and Adaptive Tumor Immunity. Semin Radiat Oncol. 2020;30(2):139-44.

16. Dar TB, Henson RM, Shiao SL. Targeting Innate Immunity to Enhance the Efficacy of Radiation Therapy. Front Immunol. 2018;9:3077.

17. Rodriguez-Ruiz ME, Vitale I, Harrington KJ, Melero I, Galluzzi L. Immunological impact of cell death signaling driven by radiation on the tumor microenvironment. Nat Immunol. 2020;21(2):120-34.

18. Schaue D. A Century of Radiation Therapy and Adaptive Immunity. Front Immunol. 2017;8:431. 
19. Moquet J, Higueras M, Donovan E, Boyle S, Barnard S, Bricknell C, et al. Dicentric Dose Estimates for Patients Undergoing Radiotherapy in the RTGene Study to Assess Blood Dosimetric Models and the New Bayesian Method for Gradient Exposure: BIOONE; 2018. 596-604, 9 p.

20. Livak KJ, Schmittgen TD. Analysis of relative gene expression data using real-time quantitative PCR and the 2(-Delta Delta C(T)) Method. Methods (San Diego, Calif). 2001;25(4):402-8.

21. Benjamini Y, Yekutieli D. The Control of the False Discovery Rate in Multiple Testing under Dependency. The Annals of Statistics. 2001;29(4):1165-88.

22. Henriksson M, Luscher B. Proteins of the Myc network: essential regulators of cell growth and differentiation. Adv Cancer Res. 1996;68:109-82.

23. Casey SC, Baylot V, Felsher DW. The MYC oncogene is a global regulator of the immune response. Blood. 2018;131(18):2007-15.

24. Elgueta R, Benson MJ, de Vries VC, Wasiuk A, Guo Y, Noelle RJ. Molecular mechanism and function of CD40/CD40L engagement in the immune system. Immunol Rev. 2009;229(1):152-72.

25. Vonderheide RH. CD40 Agonist Antibodies in Cancer Immunotherapy. Annu Rev Med. 2020;71:47-58.

26. Martin CE, Spasova DS, Frimpong-Boateng K, Kim H-O, Lee M, Kim KS, et al. Interleukin-7 Availability Is Maintained by a Hematopoietic Cytokine Sink Comprising Innate Lymphoid Cells and T Cells. Immunity. 2017;47(1):171-82.e4.

27. Barata JT, Durum SK, Seddon B. Flip the coin: IL-7 and IL-7R in health and disease. Nature immunology. 2019;20(12):1584-93.

28. Mukherjee D, Coates PJ, Lorimore SA, Wright EG. Responses to ionizing radiation mediated by inflammatory mechanisms. The Journal of pathology. 2014;232(3):289-99.

29. Vacchelli E, Vitale I, Tartour E, Eggermont A, Sautes-Fridman C, Galon J, et al. Trial Watch: Anticancer radioimmunotherapy. Oncoimmunology. 2013;2(9):e25595.

30. Meziani L, Mondini M, Petit B, Boissonnas A, Thomas de Montpreville V, Mercier O, et al. CSF1R inhibition prevents radiation pulmonary fibrosis by depletion of interstitial macrophages. Eur Respir J. 2018;51(3).

31. Kaplanski G. Interleukin-18: Biological properties and role in disease pathogenesis. Immunol Rev. 2018;281(1):138-53.

32. Xiao M. The Role of Proinflammatory Cytokine Interleukin-18 in Radiation Injury. Health Phys. 2016;111(2):212-7.

33. Reits EA, Hodge JW, Herberts CA, Groothuis TA, Chakraborty M, Wansley EK, et al. Radiation modulates the peptide repertoire, enhances $\mathrm{MHC}$ class I expression, and induces successful antitumor immunotherapy. J Exp Med. 2006;203(5):1259-71.

34. Sen GC, Sarkar SN. The interferon-stimulated genes: targets of direct signaling by interferons, doublestranded RNA, and viruses. Curr Top Microbiol Immunol. 2007;316:233-50.

35. Vesely MD, Kershaw MH, Schreiber RD, Smyth MJ. Natural innate and adaptive immunity to cancer. Annu Rev Immunol. 2011;29:235-71. 
36. Balázs K, Kis E, Badie C, Bogdándi EN, Candéias S, Cruz Garcia L, et al. Radiotherapy-Induced Changes in the Systemic Immune and Inflammation Parameters of Head and Neck Cancer Patients. 2019;11(9):1324.

37. Frey B, Mika J, Jelonek K, Cruz-Garcia L, Roelants C, Testard I, et al. Systemic modulation of stress and immune parameters in patients treated for prostate adenocarcinoma by intensity-modulated radiation therapy or stereotactic ablative body radiotherapy. Strahlenther Onkol. 2020.

38. Teresa Pinto A, Laranjeiro Pinto M, Patrícia Cardoso A, Monteiro C, Teixeira Pinto M, Filipe Maia A, et al. lonizing radiation modulates human macrophages towards a pro-inflammatory phenotype preserving their pro-invasive and pro-angiogenic capacities. Scientific Reports. 2016;6:18765.

39. Wherry EJ, Blattman JN, Murali-Krishna K, van der Most R, Ahmed R. Viral persistence alters CD8 Tcell immunodominance and tissue distribution and results in distinct stages of functional impairment. J Virol. 2003;77(8):4911-27.

40. Woroniecka K, Chongsathidkiet P, Rhodin K, Kemeny H, Dechant C, Farber SH, et al. T-Cell Exhaustion Signatures Vary with Tumor Type and Are Severe in Glioblastoma. Clin Cancer Res. 2018;24(17):4175-86.

\section{Figures}


A

B
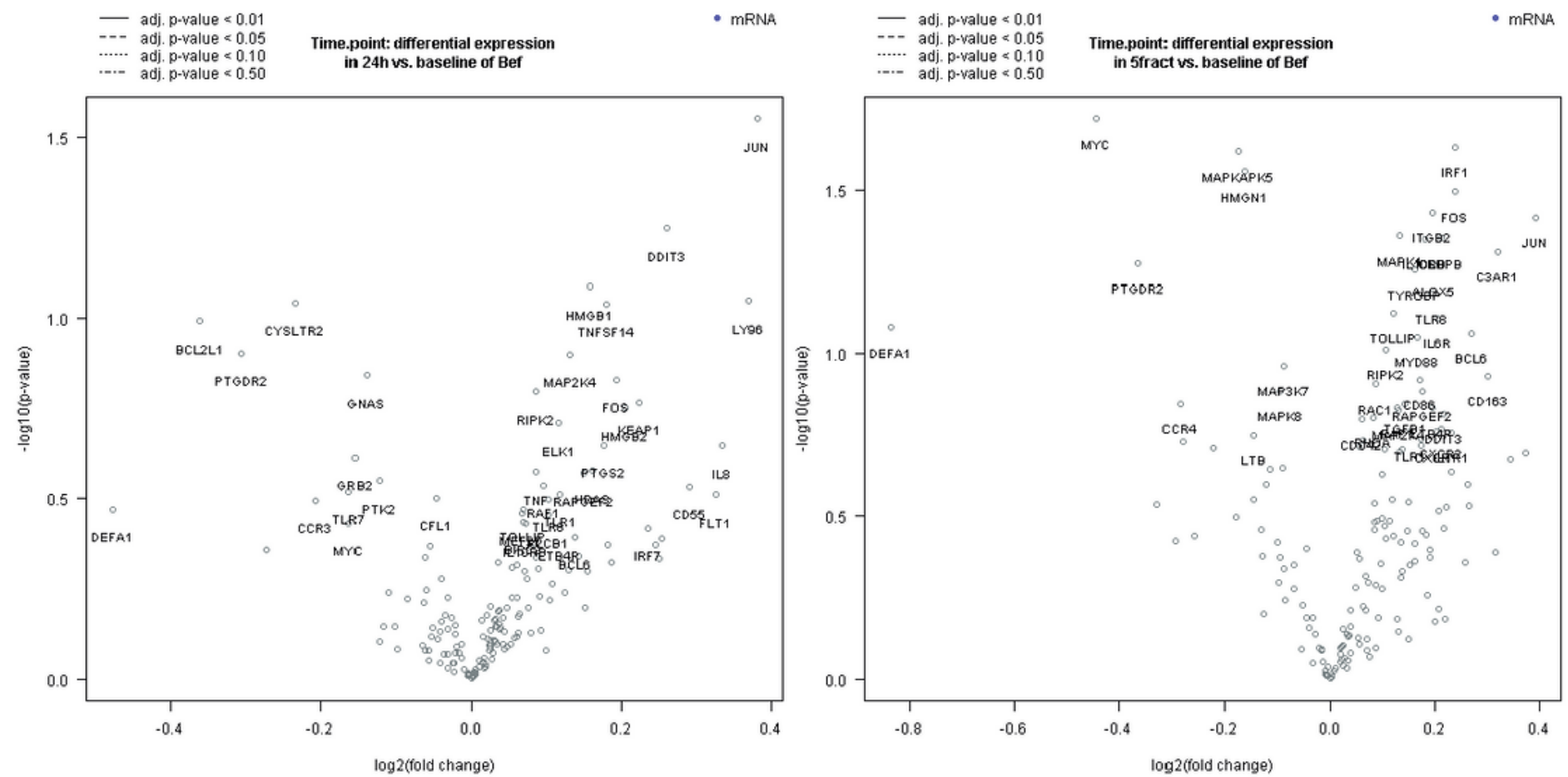

C

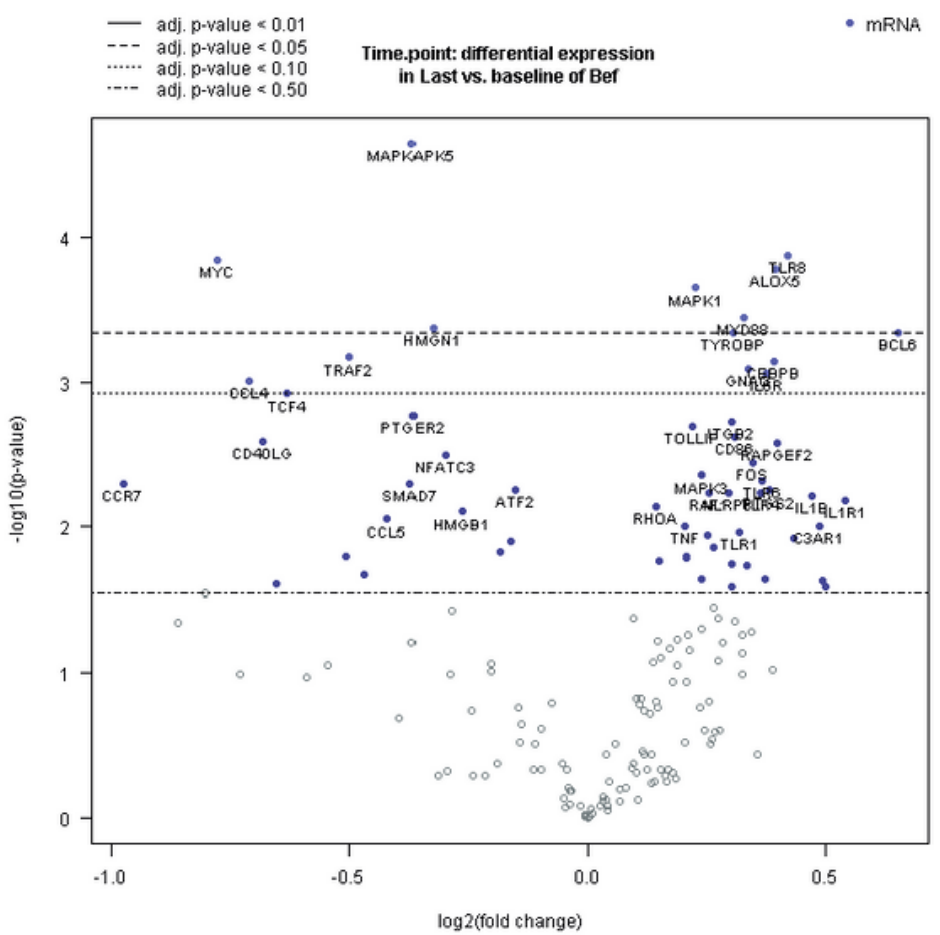

Figure 1

Volcano plots displaying differential gene expressed genes between the time points "before the start of the treatment" and (A) $24 \mathrm{hr}$ after first fraction (B) before 5-6th faction (C) before last fraction. Horizontal lines indicate various $p$-value thresholds. Genes are coloured if the resulting $p$-value is below the given $p$ value threshold. The 40 most statistically significant genes are labelled in the plot. 

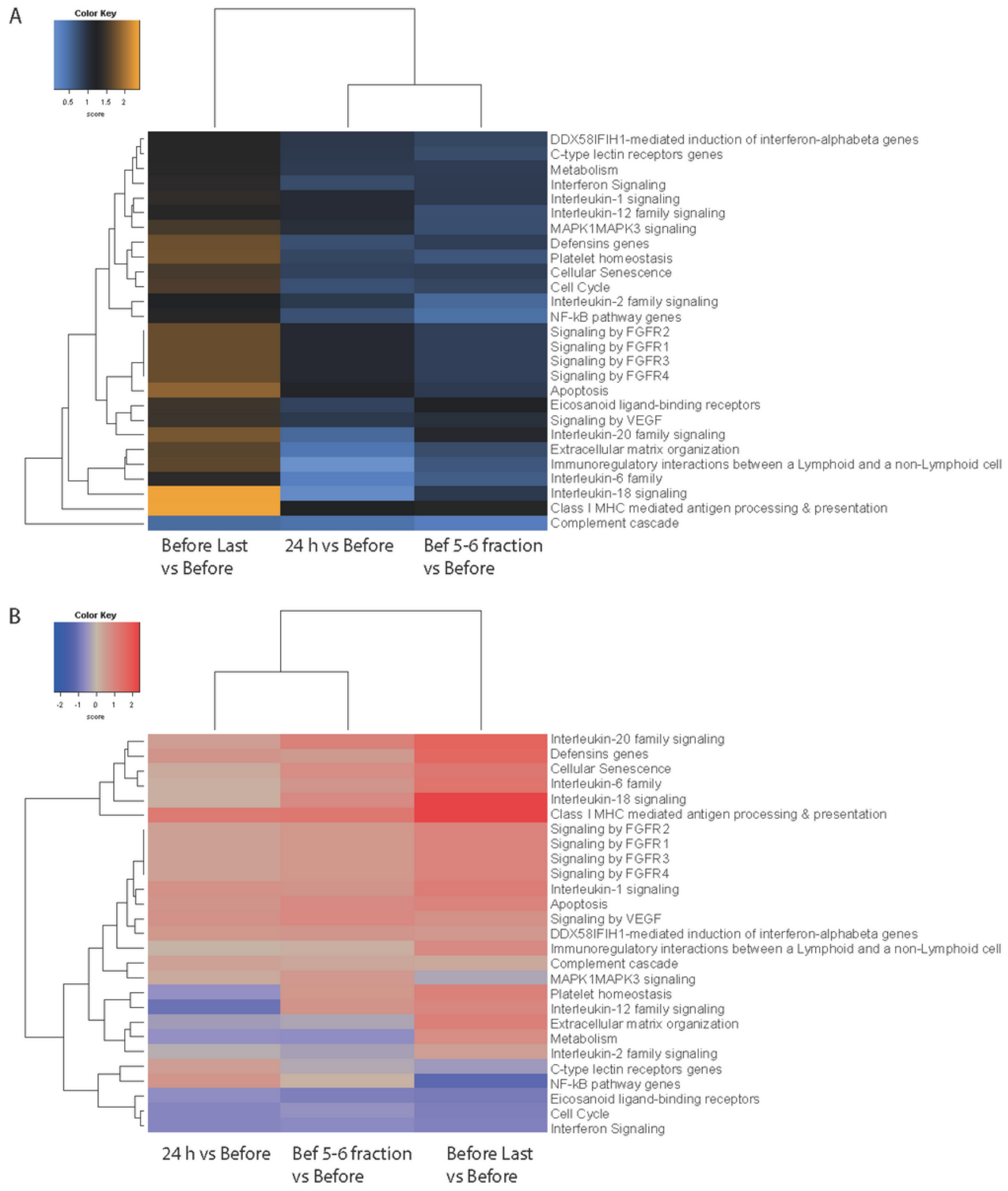

Figure 2

Figure GSA: Gene set analysis with global significance scores and directed global significance scores. (A) Global significance score plot: orange denotes gene sets whose genes exhibit extensive differential expression with the covariate, blue denotes gene sets with less differential expression. (B) Directed global significance score plot: Red denotes gene sets whose genes exhibit extensive over-expression with the covariate, blue denotes gene sets with extensive under-expression. 


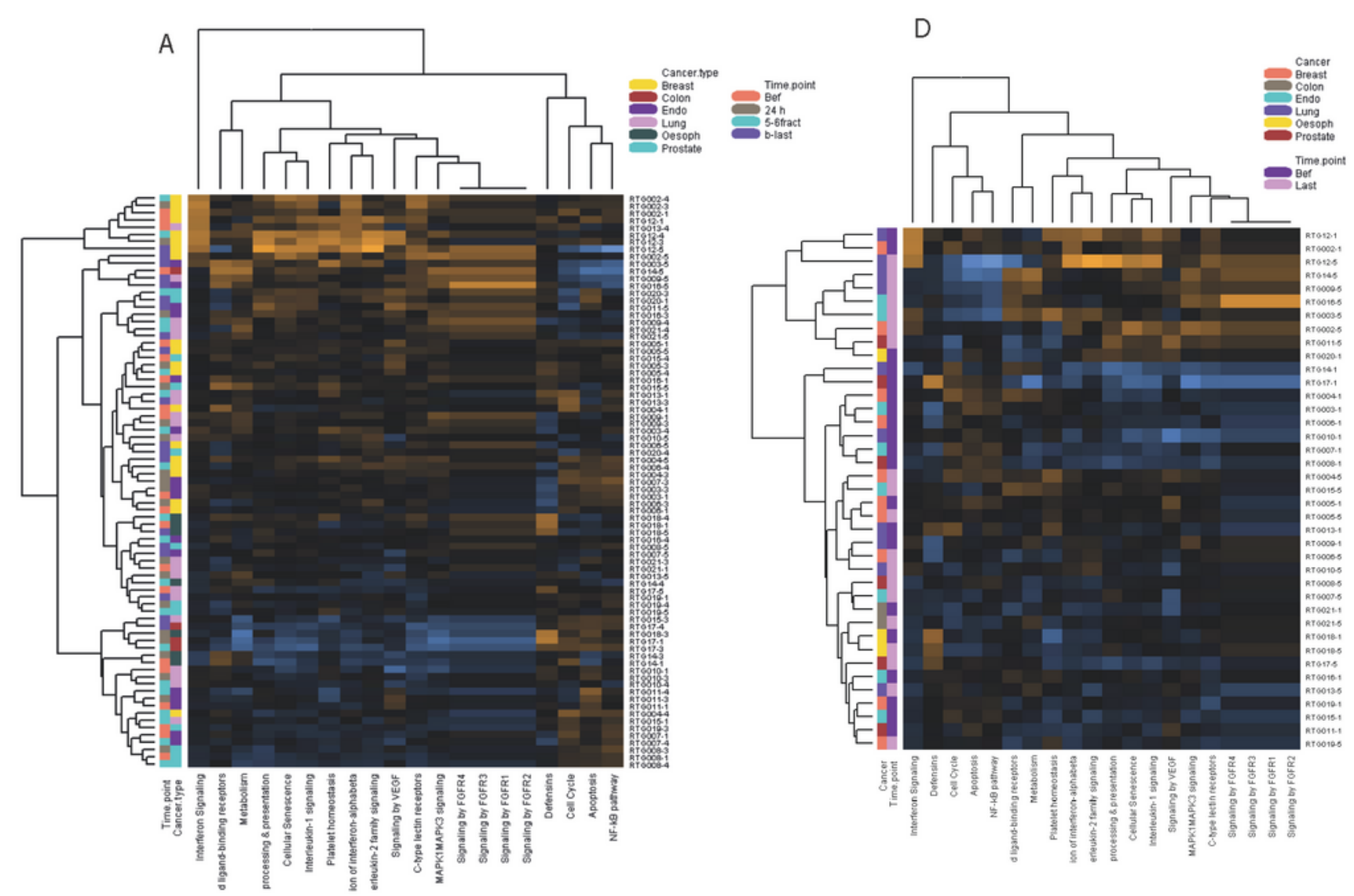

B
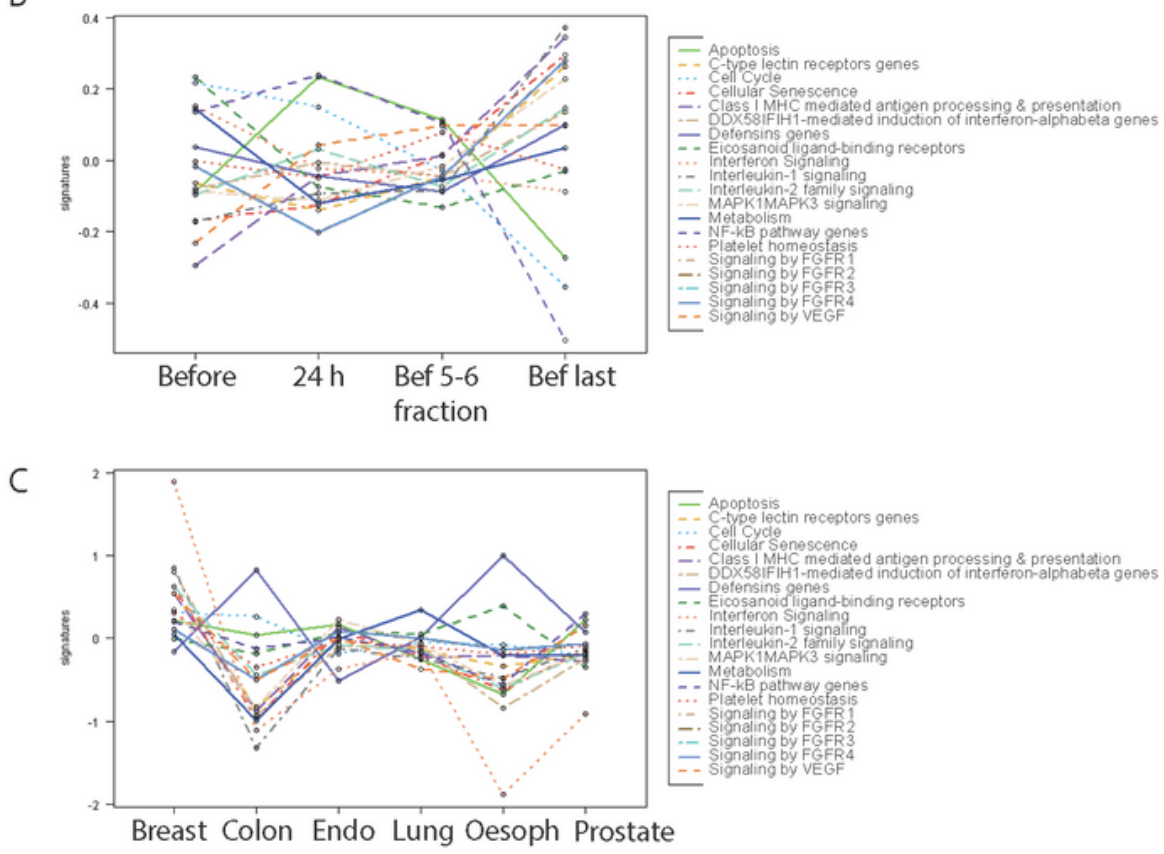

\section{Figure 3}

Pathway changes in the different time points (A) Heatmap of pathways scores including the 4 time points and the 6 cancer types: Orange indicates high scores; blue indicates low scores. Scores are displayed on the same scale via a Z-transformation. (B) Individual pathway scores for each time point (C) Individual pathway scores for each cancer type. (D) Heatmap of pathways scores comparing the time point "before" and "before last fraction" together with the 6 cancer types. 


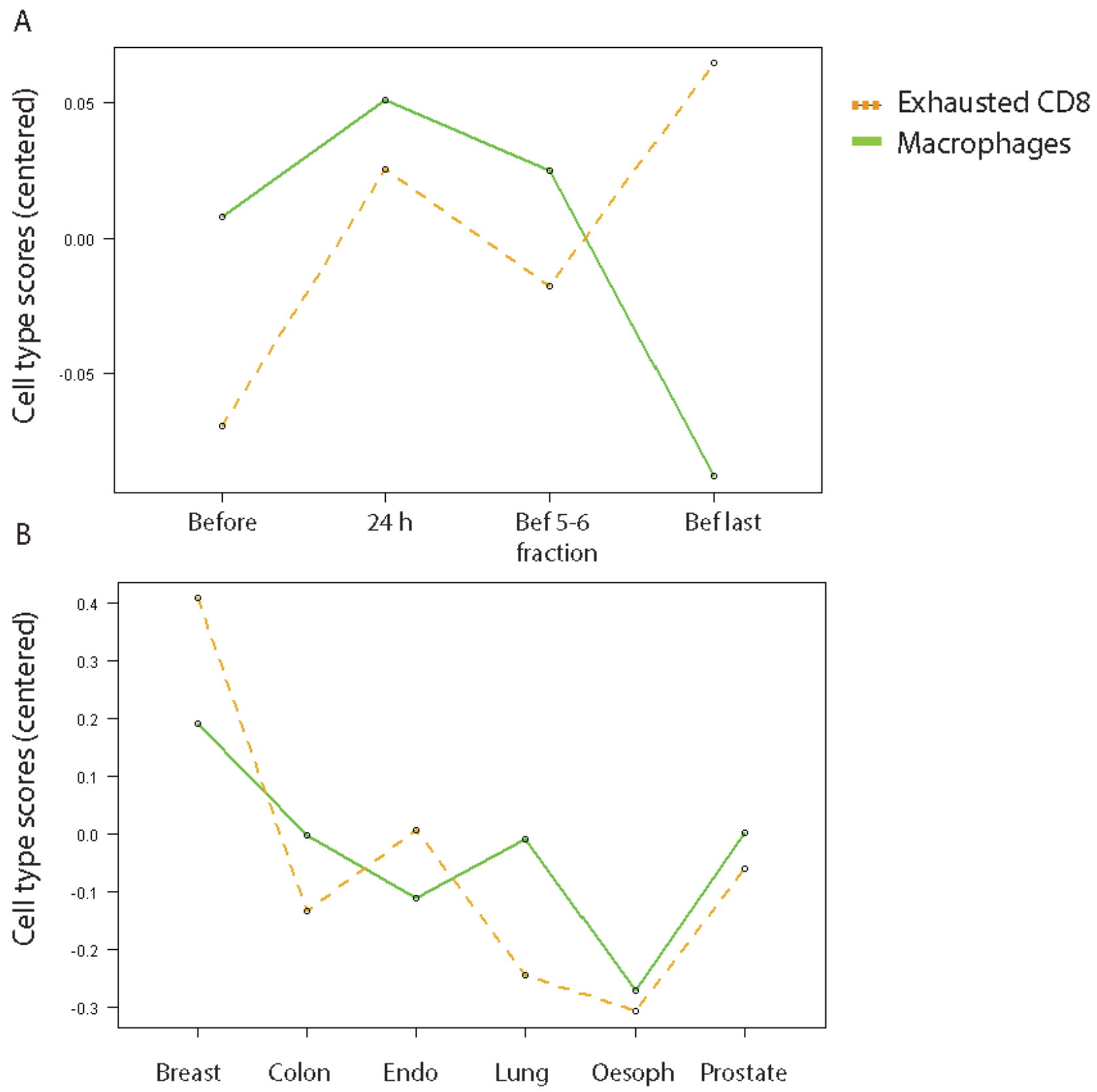

\section{Figure 4}

Immune cell profiling analysis indicating cell type abundance measurements versus time points (A) or versus cancer type at all time points (B). Raw cell type measurements are calculated as the log2 expression of each cell type's marker genes. 


\section{nCounter}

qPCR

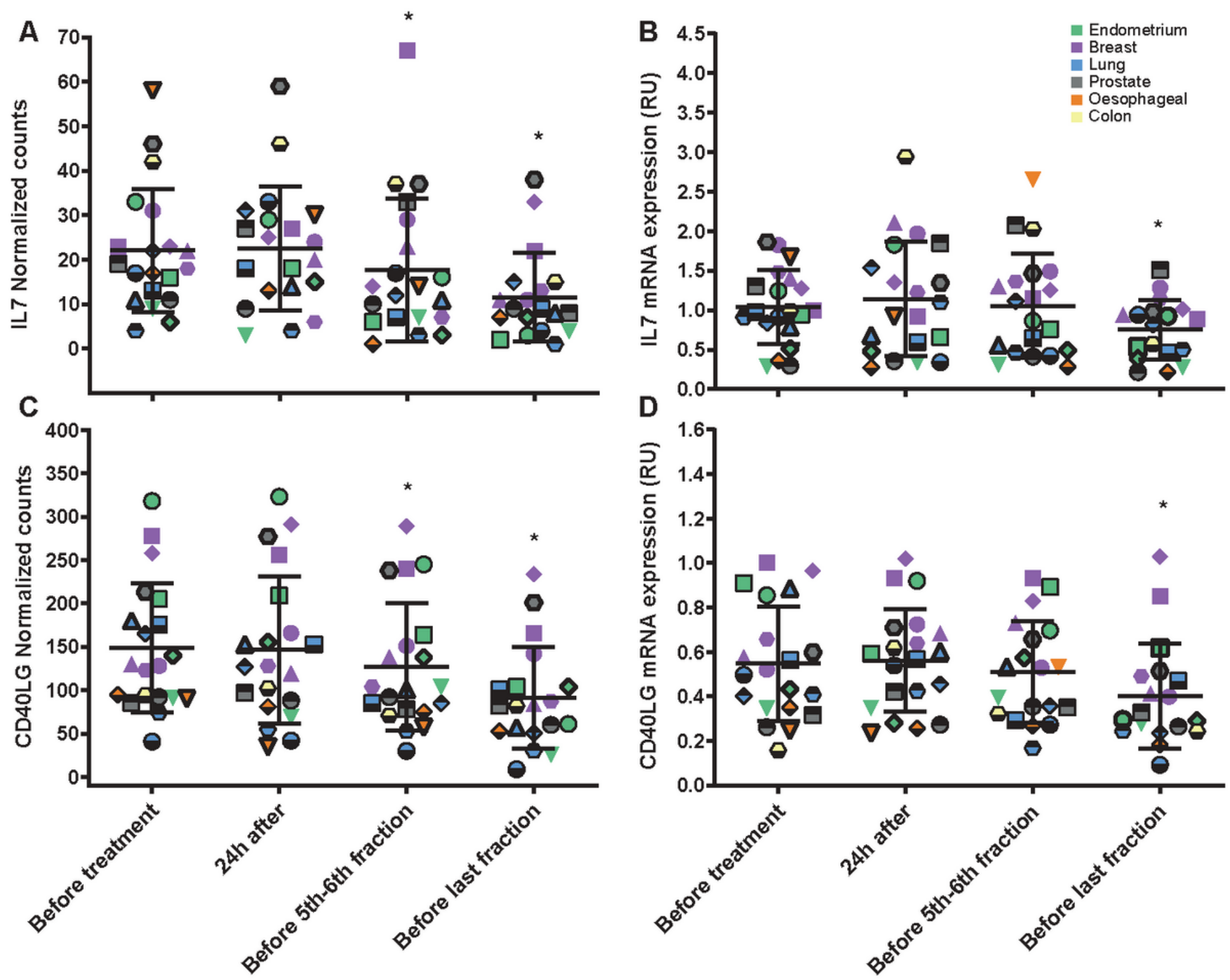

Figure 5

mRNA expression levels of IL7 and CD40LG in blood from radiotherapy patients analysed by nCounter analysis (panel A and C) and APCR (panel B and D). Blood samples from 20 patients, comprising those with endometrial, breast, lung, prostate, oesophageal and colon cancer, were analysed. Blood was collected at four time points: before the start of the treatment, at $24 \mathrm{~h}$ after the first fraction, before 5th6th fraction and before the last fraction. Individual data points are shown for all patients, together with the mean $\pm S D$ (each patient is represented with a different symbol). Each cancer group was color coded. Statistical analyses were performed in log-transformed data. *Significantly different from the control (before treatment) (paired $t$ test, $P \leq 0.05$ ). 